\title{
Interactive Storytelling with Virtual Identities
}

\author{
M. Greeff \\ CSIR: icomtek, Scientia \\ Pretoria 0001, South Africa \\ mgreef@csir.co.za \\ V. Lalioti \\ Computer Science Department, University of Pretoria \\ Pretoria 0001, South Africa \\ vlalioti@cs.up.ac.za
}

\begin{abstract}
Stories form an integral part of our lives. Interactive storytelling enables the participant to actively explore the story world. However, interactive storytelling has problems such as the problem of narrative flow, the character's knowledge of the world, internal consistency, time and ease of creation. We introduce the concept of using virtual identities for interactive storytelling in virtual environments and discuss how this approach solves some of the problems that are encountered with interactive storytelling. We discuss authoring tools and how we used the AVANGO framework to develop our application and the results that were achieved when the application was tested on the monitor and in Cyberstage.
\end{abstract}

\section{Introduction}

Since our childhood we are exposed to stories in many different forms. Legends and stories about our culture and heritage are passed on from generation to generation. The Story metaphor has been used in Multimedia and Virtual Environments to create interactive stories. Earlier systems used video, videodiscs or the television to create a non-linear story, in which the user can make a choice between different predefined paths at certain points in the story. Virtual environments are much richer both in terms of freedom of navigation and ease of interaction. In projection-based systems in particular, the user is not bound to predefined paths and can have a hands-on experience through immersion and interaction with the virtual world. However guidance is also needed in these environments in order to support the user during the exploration and facilitate the understanding of the purpose and intention of the particular virtual environment.

Interactive stories have already been used in Virtual Environments and allow the users to be actively involved in the story, since they can easily relate to it and experience joy while engaging in the interactive story world [2] [3] [12]. Although interactive storytelling gives the participant a richer experience, a number of aspects are missing from today's approaches, which relate among others to the narrative flow 
and internal consistency of the story. Another important issue is the ease of creation of interactive stories for virtual environments. In this paper we investigate these issues and present an approach for authoring interactive stories in virtual environments that address some of these aspects.

Our approach allows for the creation of several virtual identities through the eyes of which a user can experience the virtual world. Each identity is empowered with knowledge about itself, its perception about the virtual environment and its embodiment in the virtual world. Therefore the user can experience the same virtual world through different virtual identities being immersed each time in a different interactive story. The approach allows free navigation and interaction with respect to the particular virtual identity used. Furthermore, transitions from one virtual identity to another are possible and allow the creation of a more complex interactive story. We developed a framework and an authoring tool for defining and creating these identities, which are presented in this paper in detail. The authoring tool and the approach have been tested in a Cultural Heritage application and demonstrated in Cyberstage - GMD's surround-screen projection-based stereoscopic display system and the results are also presented in this paper.

\section{Background}

The traditional methods of lectures, print, audio and video are narrative media, because they require a storyline and put the teacher in the role of the storyteller and the learner in the role of the listener. Interactive stories let the story world come alive and give the participant much more freedom. They are particularly suited for facilitated exploration and guidance in virtual environments, since they can incorporate rich interaction and free exploration, taking advantage of all the benefits and power of these environments to create a much richer experience for the user.

Although interactive storytelling gives the participant more freedom, a number of aspects relating to the character knowledge of the virtual world, narrative flow, the internal consistency of the story, the handling of time and finally the ease of creation can limit the benefits from its use in virtual environments [4] [5]. Taking a closer look at each of the aspects mentioned above reveals the following:

\section{Character's Knowledge of the Story World}

In a typical, non-interactive story, the characters understand the world and have knowledge about their world, for example a character knows the contents of the drawers and closets in her apartment and there is no need to open them to see what they contain. But in an interactive story world, the user does not have this knowledge and needs to explore the different objects as if she was a total stranger to the world. This approach can be used for the creation of interactive stories in some virtual environments, like in mysteries and heroic quests where the user will engage in new and unfamiliar worlds. However, in many applications of virtual environments it is important to provide more guidance and share some of the character's knowledge with the user. For example, in applications of virtual environments for education and 
cultural learning, knowledge about the story world should be incorporated in the interactive story so that the user can acquire it through this facilitated interactive experience.

\section{Internal consistency}

Any story must be coherent; that is, at any point in the story the circumstances at that specific point must be consistent with everything that happened beforehand. This does not mean that stories must be predictable, rather that they should make sense in a satisfying manner. However, the core of interactivity is freedom and therefore a conflict arises between the user's desire to do as he chooses and the story world creator's desire to impose a plot and characterisation on the user. The difficulty with using interactive storytelling in virtual environments is to ensure that a high degree of user freedom is combined with the story in a coherent way at every instance of the virtual experience.

\section{Narrative flow}

Traditionally, every story consists of an introduction, rising action, a climax, falling action and a conclusion. With non-interactive stories this can be accomplished because the author is in complete control of the story and the characters. With interactive stories the user is outside the control of the author and therefore it is very difficult to ensure that when the dramatic climax takes place in the story, the user is there and ready for it. This is known as the Problem of Narrative Flow [4].

Adventure games try to solve this in a number of ways:

- Limiting the interactivity by either cutting down the interactivity so that the user cannot stray from the plot or giving the user a lot of interactivity that is not necessarily meaningful but does not affect the plot. This solution is obviously taking away some of the power that interactive storytelling can give to the user.

- Not taking the user's readiness for the climax into consideration - the story world is alive and exists around the user but regardless of what he is doing. Therefore, in this solution, the user is not allowed to influence the story through any interaction.

- Advancing the plot along with the user's advances - the user's actions are linked to the advancement of the plot and therefore this approach absolutely guarantees that the user is ready for the climax. However, this solution is mechanistic and the story only progresses when the user performs the right actions.

\section{Time}

In traditional narrative, the story and the discourse are divided. When reading a novel, the storyline is constructed from the discourse that it is presented. Therefore, time of the narrated, time of the narration and time of the reading do not necessarily coincide. 
Christian Metz, as quoted in [7], says that:

"Narrative is a ... double temporal sequence... : There is a time of the thing told and a time of the narrative (the time of the signified and the time of the signifier). This duality not only renders possible all the temporal distortions that are commonplace in narratives (three years of the hero's life summed up in two sentences of the novel or in a few shots of a "frequentative" montage in film, etc.). More basically, it invites us to consider that one of the functions of narrative is to invent one time scheme in terms of another time scheme."

It is obvious that presenting time in an interactive story becomes more complex, because of the interactive nature of the story. The representation of time and transitions in time is a particular important challenge in virtual environments were the time of the virtual experience can vary for different users or even for the same user during the repetitive use of the virtual environment.

\section{Ease of creation}

One of the main problems with using interactive storytelling in virtual environments is the difficulty in creating an interactive story. Most writers and storytellers have little or no knowledge of programming. On the other hand, the existing authoring tools for virtual environments are very specialised and require a user experienced in programming. Authoring tools are generally defined as tools for creating computerbased instruction without having to program [8]. Authoring tools for interactive storytelling should allow writers to concentrate on creating the story instead of the details of programming.

An example of an authoring tool is the Erasmatron [9]. It was developed to enable non-technical writers to create interactive stories without worrying about technical details, and to address the problem of exploding nodes when the choose-your-ownadventure approach is followed. It has editors, navigational aids and rehearsal to help the user create an interactive story world.

Authoring tools use metaphors such as paths, scenes and scripts to describe what the user experiences and what interaction is allowed with the environment. Therefore, authoring tools provide solutions to make the creation of interactive story worlds easier. They provide some internal consistency by defining what interaction is allowed in the story world, and ensure consistency using for example predefined paths. Predefined paths can also provide a solution for the Problem of Narrative Flow, but are still limiting the virtual experience. In addition, the problem of time and knowledge about the world remains unsolved and the user experiences the story world from only one angle, that of the specific script and path.

\section{Virtual Identities Authoring Approach}

In [1] we proposed the virtual identity approach to interactive storytelling. With this approach the participant experiences the interactive story through the eyes of a virtual identity. Each virtual identity is defined by the knowledge about itself, its perception 
about the environment and its virtual embodiment. We extended this approach by providing a framework to be used for defining the virtual identity, with the following main features:

- Characteristics that a virtual identity is born with

- Characteristics concerning the virtual identity's background

- Embodiment of the virtual identity

- Behavioural characteristics of the virtual identity

An initial approach that relates real identities to virtual identities, in terms of sociopsychological, gender and embodiment issues, presented in [1] is extended in this paper and the taxonomy (framework) will be presented in the full paper, in this section. Our authoring tool is based on this framework and it is fully described in section 4 with the authoring of an application in the Cultural Heritage area.

The Virtual Identities Authoring approach provides solutions to the problems mentioned in the introduction. We will now take a closer look at the solution for each of the problems mentioned in section 2.

\section{Knowledge about the world}

Each virtual identity is empowered with knowledge about itself, which it uses to perceive and interact with the virtual world. Therefore, although the virtual identity does not have knowledge about the story world, it does have knowledge about itself, for example its cultural background, age and gender. This partially defines its perception of the world and allows different interactions to the virtual world for each identity.

\section{Internal consistency}

Each virtual identity is allowed certain interactions with the virtual world according to its knowledge about itself. Therefore, all the user actions are coherent and go along with the story. By following this approach, internal consistency is maintained.

\section{Problem of Narrative Flow}

In our approach, the story unfolds as the user explores and interacts with the world, through the embodiment of a virtual identity. The interactive story created by this experience is not one with a specific climax and therefore the narrative flow problem is avoided.

\section{Time}

To allow change in time, we introduced transitions, both forward and backward in time. This will result either the change from one identity to another or the change of the age characteristic of the same identity. When the user interacts with an object in the virtual world that brings back memories, then a temporary change in the age characteristic of the virtual identity will result for example a flashback on its childhood, or the lowering of the height and thus the point of view of the user. In the 
second case, a transition from a child virtual identity to an adult identity results in a first experience through a child's eyes and then through the adult's.

\section{Authoring Tool}

An authoring tool enables users to create interactive stories. With our approach, the user of the authoring tool is able to create many different experiences with the use of a single model. Each virtual identity is defined by giving values to certain characteristics as described in section 3. Furthermore, certain interactions with the virtual environment are allowed in respect to these characteristics. In addition, the virtual embodiment of the identity can take many forms - i.e. video, avatar, sound, voice - which also automatically defines issues of navigation and interaction. For example, a virtual identity embodied in an avatar is not allowed to go through walls, while a voice is not allowed to grab certain objects, but can only talk about them. This approach and the inclusion of transitions in time allow a participant to have many different experiences with the same model of virtual environment (story world), and thus considerably reducing the authoring effort.

We developed the authoring tool with the AVANGO [6] framework that has been under development at GMD since 1996. AVANGO provides programmers with a scene-graph that is accessible from all the processes in the distributed application. The $\mathrm{C}++$ programming language is used to define nodes or sensors (two categories of object classes). AVANGO also features a complete language binding to the interpreted language, Scheme, which is a high-level language that supports operations on structured data, e.g. strings, lists and vectors.

We tested our idea in a Cultural Heritage application. The story world is a shebeen (township tavern) in Cato Manor [10]. Cato Manor was once a vibrant Southern African community with Indian and African cultures, but in the 1950's the cumulative effects of overcrowding, deteriorating living conditions, hostility and neglect, lead to a wave of interracial violence and rebellion against the establishment. During the darkest days of apartheid it was torn down to enforce racial segregation and open up a prime piece of real estate for white occupation.

We used a 3 dimensional model of a shebeen in Cato Manor [10] developed by CSIR, under the Cultureware ${ }^{-1}$ project. The model was enhanced and imported to AVANGO, by using the hook mechanism for the objects of interest. We created three identities, namely a shebeen owner, a Zulu man and a Zulu boy. By using the same

\footnotetext{
${ }^{1}$ CSIR is The South African Council for Scientific and Industrial Research. The Cultureware project is fully funded under DACTS (National Department for Arts, Culture, Science and Technology of South Africa) through the Innovation Fund Programme. http://www.cultureware.net
} 
model (the shebeen) and allowing certain interactions in the shebeen according to the identity, the user can explore the shebeen through the eyes of each identity.

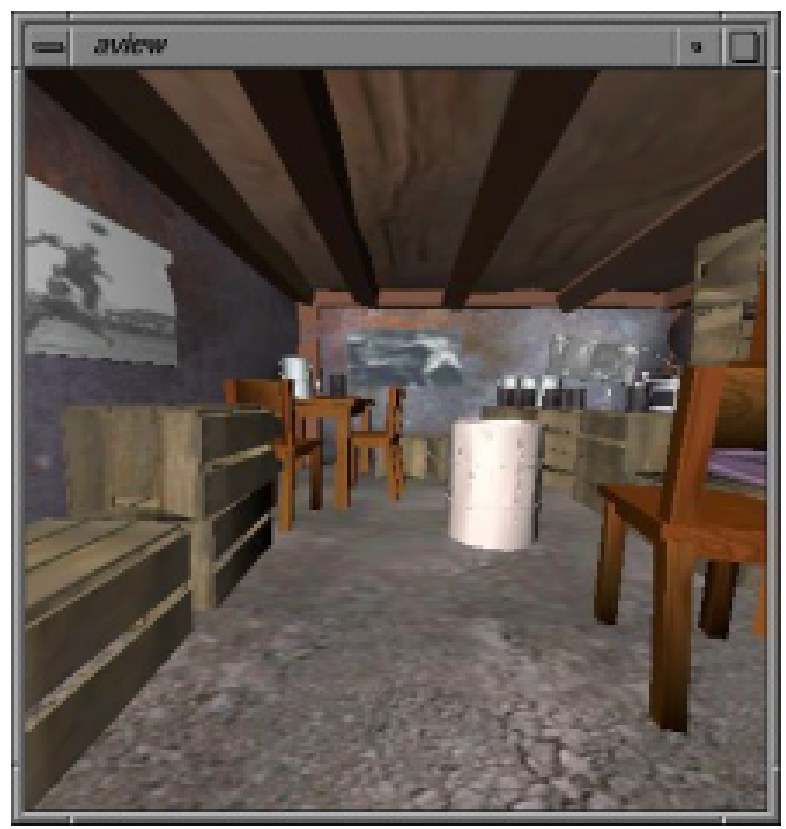

Fig. 1. The model of a shebeen (township tavern)

According to the virtual identity's age, the viewpoint of the identity as s/he explores the shebeen will be changed to provide the user with a realistic perspective of the shebeen. The Zulu boy's viewpoint is much lower than that of the Zulu man and is illustrated in figure 1 .

The Zulu man is allowed to move the crates and chairs and to drink beer from the cups. The Zulu man can also click on one of the photos in the shebeen, which will then trigger a sound file to be played. The Zulu boy is allowed to also click on one of the photo's and can move the cups around, but he is not allowed to drink from the cups. Therefore, different cultural experiences can be obtained, allowing a rich cultural learning experience. We have also demonstrated this application in Cyberstage with very promising results. 


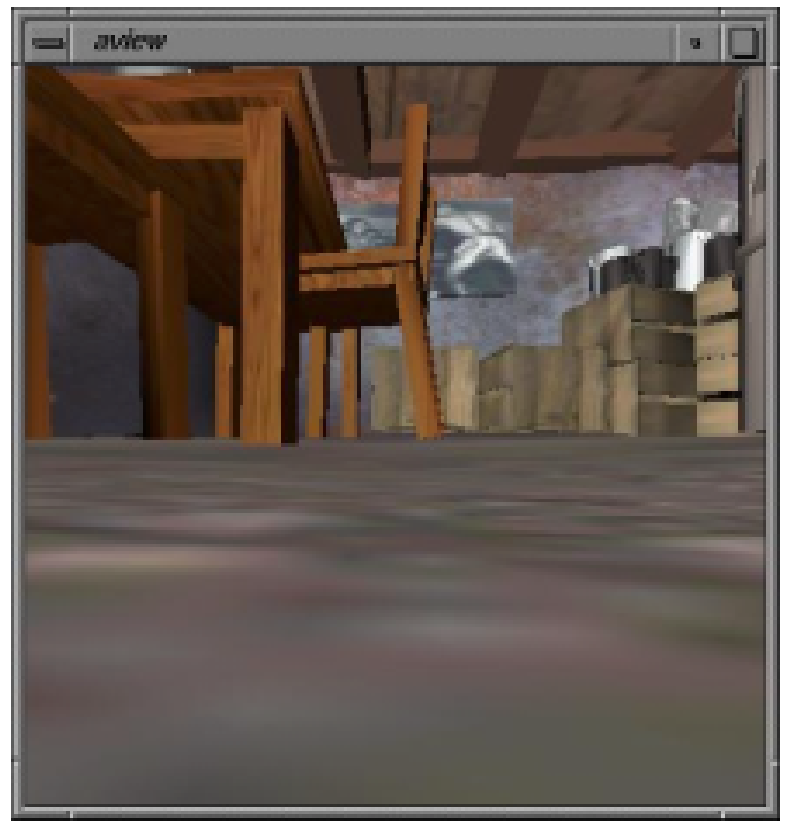

(a) Zulu boy's viewpoint in the shebeen

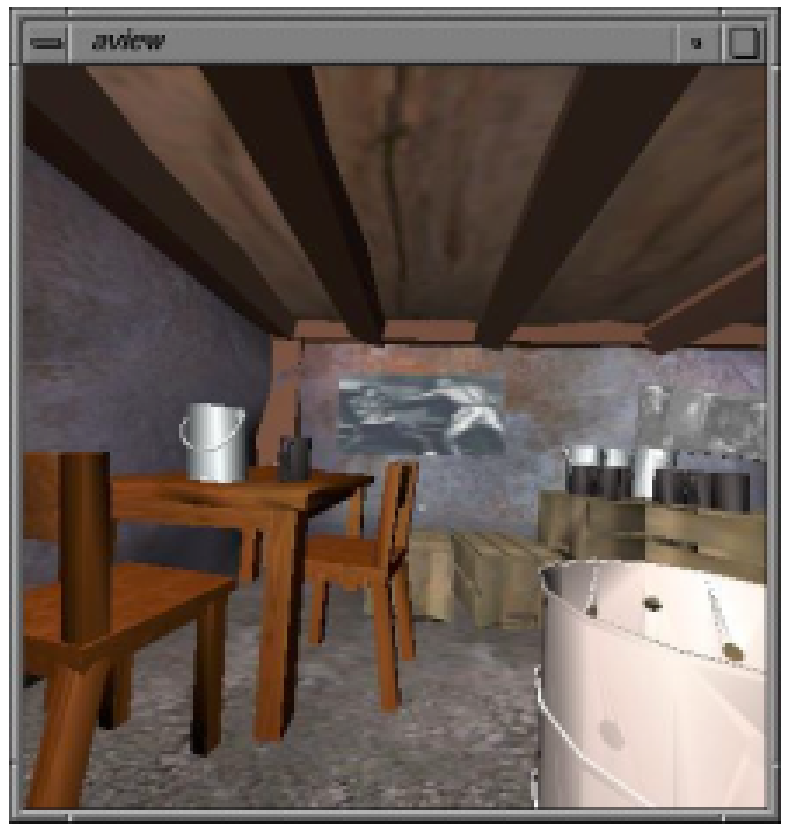

(b) Zulu man's viewpoint in the shebeen

Fig. 2. Illustration of the difference in viewpoint of the Zulu man from the Zulu boy 


\section{Conclusions}

In this paper we presented an approach and an authoring tool for interactive storytelling in virtual environments using the virtual identities method. In this approach, the user can have a realistic and multiple rich experiences through the eyes of the different identities. We have implemented one application in the area of cultural heritage and demonstrated it in Cyberstage - GMD's surround-screen projection-based stereoscopic display system. This approach can also be used in many application areas, such as entertainment, culture and education. We are currently improving the User Interface of the authoring tool and further extending the virtual identity approach and framework.

\section{Acknowledgements}

We would like to thank C. Jackson from CSIR for her valuable input on issues of social psychology, gender and culture and CSIR for allowing us to use the sheebeen model. Special thanks to all the members of GMD's Virtual Environments group. With their support and valuable help this work was made possible.

\section{References}

1. Jackson, C., Lalioti, V.: Virtual Cultural Identities, CHI-SA 2000, South African Human-Computer Interaction Conference 2000, University of Pretoria Conference Centre, Hatfield, South Africa, May 8-10, 2000.

2. Johnson, A., Moher, T., Ohlssoon, S., Gillingham, M.: The Round Earth projectcollaborative VR for conceptual learning, IEEE Computer Graphics and Applications, Virtual Reality, Vol. 19, No. 6, Nov/Dec 1999.

3. The MIT Media Lab, KidsRoom: Action Recognition in an Interactive Story Environment, October 1996. http://vismod.www.media.mit.edu/vismod/demos/kidsroom/

4. Adams, E. (Gamasutra): Three Problems for Interactive Storytellers, December 1999.

http://www.gamasutra.com/features/designers_notebook/19991299.htm

5. Juul, J.: A Clash between Game and Narrative, First International SKIKT Conference on Digital Arts and Culture, Bergen, Norway, November 26-28, 1998.

6. Tramberend, H.: AVANGO: A Distributed Virtual Reality Framework, In Proceedings of the IEEE Virtual Reality '99, JW Marriott Hotel, Houston, Texas, USA, March 13-17, 1999.

7. Genettes, G.: Narrative Discourse, Cornell University Press, 1980, p. 33.

8. Locatis, Ullmer, Carr, Banvard, Le, Lo, \& Williamson: Authoring system reassessed, Educational Technology Research and Development, Vol. 40, No. 2, p. 77-82, 1992. 
9. Crawford, C.: Erasmatazz, www.erasmatazz.com

10. Lynch, B.: Cato Manor Development Project, http://www.cmda.org.za

11. Hu, J.: Spatial ability and hypermedia authoring: The attitudes of novice hypermedia developers and the characteristics of their concept maps and Authorware projects, http://nt-hsta.hsc.wvu.edu/health/personal/AERAProposal.html

12. Bobick, A., Intille, S., Davis, J., Baird, F., Pinhanez, C., Campbell, L., Ivanov, Y., Schütte, A., Wilson, A.: The KidsRoom: A perceptually-based interactive and immersive story environment, PRESENCE: Teleoperators and Virtual Evironments, 8(4), August 1999, p. 367- 391. 